\title{
Mental Workload of Local vs Remote Operator in Human-Machine Interaction Case Study
}

\author{
Melanie ZIMMER ${ }^{\mathrm{a}, 1}$, Ali AL-YACOUB ${ }^{\mathrm{a}}$, Pedro FERREIRA ${ }^{\mathrm{a}}$, Ella-Mae HUBBARD ${ }^{\mathrm{a}}$ \\ and Niels LOHSE ${ }^{\text {a }}$ \\ ${ }^{a}$ Loughborough University, Intelligent Automation Centre, Epinal Way, Loughborough \\ LE11 3TU, UK
}

\begin{abstract}
Since late 2019, a novel Coronavirus disease 2019 (COVID-19) has spread globally. As a result, businesses were forced to send their workforce into remote working, wherever possible. While research in this area has seen an increase in studying and developing technologies that allow and support such remote working style, not every sector is currently prepared for such a transition. Especially the manufacturing sector has faced challenges in this regard. In this paper, the mental workload of two groups of participants is studied during a human-robot interaction task. Participants were asked to bring a robotised cell used in a dispensing task to full production by tuning system parameters. After the experiment, a self-assessment of the participants' perceived mental workload using the NASA Task Load Index (NASA-TLX) was used. The results show that remote participants tend to have lower perceived workload compared to the local participants.
\end{abstract}

Keywords. Mental Workload, NASA-TLX, Remote Working, Ergonomics, Human-Robot Interaction.

\section{Introduction}

Under COVID-19, several industries have realised the need for remote working practices. While for some sectors the move from working locally to remotely has been swift, others have faced more challenges to do so. Remote working brings its unique challenges for the workplaces, not just for the companies but also the individuals. This holds especially true in manufacturing where particular processes, such as corrective maintenance or ramp-up, require unpredictable physical work. Any delay here can lead to significant delays in terms of time and, consequently, result in a financial loss. Under these restrictions, the idea of remote collaboration or a remote expert working with a local operator to achieve a common goal has become a more accepted one. Here, the remote experts provide their knowledge by interacting with their co-workers and systems using technology [1]. While for the local operator the focus will be more on the physical aspects of the task at hand, the remote operator's role is more the one of a decisionmaker. The quality of the decisions made by the remote expert or their general performance not only depend on their knowledge but also their mental workload (MWL). By knowing more about the MWL of the remote expert, additional aspects can be

${ }^{1}$ Corresponding Author. m.zimmer2@lboro.ac.uk 
addressed such as "How complex are the tasks that the operator is required to perform? Can any additional tasks be handled above and beyond those that are already imposed? How many people are needed to successfully carry out the task?" [2]. Therefore, operator and system performance can be predicted based on the quantified mental cost of performing tasks [3]. The impact on the cognitive overhead of a remote expert is yet understudied.

In this paper, the perceived MWL of human operators is analysed for remote and local participation after bringing an industrial robotised gluing station to full production. It is hypothesised that the task is less demanding for remote participants in terms of perceived workload than undertaking this task locally. One of the well-established ways to measure the MWL is the NASA-Task Load Index (NASA-TLX) [4], which is based on six sub-scales associated with the MWL, namely mental, physical, and temporal demand, as well as performance, effort and frustration.

The rest of the paper is structured as follows: a brief literature review is presented in Section 2. Section 3 outlines the methodology that was applied for this study, including a description of the task, recruited subjects and experimental setup. Section 4 provides the results and discussion, before Section 5 provides conclusions and an outlook for future work.

\section{Literature Review}

Due to the growing use of computerised and semi-automated technologies in both administrative and manufacturing tasks, the notion of MWL has grown in importance to address the difficult demands on the human's mental or information-processing abilities [5]. Huey and Wickens [6] have shown that high task demands lead to reduced performance, and increase in response times and errors, and a reduction in productivity as task performance strategies change. MWL is often considered as a multi-dimensional concept, which is characterised by the operator and task as well as the environmental context [2], and, as such, no single definition of the term mental workload can be found in the literature [7]. However, the number of tasks, the time required to do these tasks, as well as the subjective experiences by the human all seem to be associated aspects [8]. Under consideration of these aspects, Young and Stanton (cited in [9, Ch. 39]) describe the MWL as "the level of attentional resources required to meet both objective and subjective performance criteria, which may be mediated by task demands, external support, and past experience". To address and increase the key targets of ergonomics, such as the level of efficiency, satisfaction, safety and comfort in the workplace, assessing the MWL is a critical component in the enhancement of human-machine interfaces [10].

In a local and remote operator setting, the local operator is immersed in the environment the problem occurs while also having the ability to undertake physical changes, in contrast to the remote operator who is more knowledgeable in terms of how to address the problem under consideration [11]. There are different ways how both operators can work together to achieve their common goal. In terms of a remote expert and local operator working collaboratively, this can simply be done in the form of conversations and information exchange of two workers working on an assembly task [12]. Those conversations mainly identify the tasks' goal, the instruction for tasks, and the tasks' completion. Further studies introduced a typical collaborative work that involves sketches and writings [13]. It has been noted that this standard view supported 
the work process and communicated information adequately. Another study tried to determine which visual information provide a benefit for a coworking team. The study by Kraut et al. [14] examined an operator's performance working alone on a bicycle repair task in comparison with a group consisting of a remote mechanic and a local operator working on the same task. The experiment presented evidence for the effect of a shared visual context in remote collaborative work. Gergle et al. [15] further studied puzzle tasks where the shared visual context was also crucial for situation awareness and a common understanding. Oftentimes, humans use gestures as an additional means to communicate their message. Thus, further research [16] suggested that communication can be still more enhanced by showing the gestures of the expert as part of the environment the operator works in instead of providing complex descriptions.

\section{Methodology}

\subsection{Task}

Participants were asked to tune a robotised Gluing Workstation (GWS) to volume production for three different products as quickly as possible, meaning using the least number of trials. It is noted that there was no limitation on how many trials could be done and this decision was solely left with the participants. After the practical part of the experiment was finished, participants were asked to fill in an online post-questionnaire to be able to capture a more subjective feedback from the participants.

\subsection{Subjects}

For this study, participants have been assigned to two different scenarios that can be described as follows:

Group A: Participants in this group took part in the experiment in the lab environment. Change actions were done by the participants themselves.

Group B: Participants in this group took part in the experiment remotely. In this case, the experiment investigator undertook the physical changes to the setup for them. Interaction between the investigator and participant were, however, kept to a minimum (e.g. technical issues).

Participants were recruited from technical and non-technical backgrounds having little to no knowledge about the setup and its behaviour. In total, 6 female participants and 10 male participants took part. Participants' age ranged from 20-29 (7x) to 30-39 $(8 \mathrm{x})$ and 60 and above $(1 \mathrm{x})$ years. Finally, 7 participants rated their experience with technological equipment very good, 7 good, 1 satisfactory, and 1 poor with the number of years worked in the field of automation or engineering ranging from 1 to 30 years.

\subsection{Experimental Setup}

The main hardware components for the GWS setup were an ABB IRB 120 6-axis industrial robot with a two-finger SCHUNK gripper to manipulate a metal workpiece. To make the dispensing process possible, an automated time-pressure dispensing unit (Fisnar JB1113N) was used, which was connected to a syringe that has been mounted to the surrounding cell and contained the dispensing material. In addition, a Raspberry Pi3 
had been fitted to the robot cell providing temperature and humidity data about the environment. The experimental setup also contained two computers, where one machine was used to run the robot control and local Graphical User Interface (GUI), and the other one hosted the GUI for remote participation where users could choose the parameter settings for the setup and any instructions to the local operator that were then both displayed to the local operator through the local GUI. To ensure that remote participants would have the best possible and realistic experience, four cameras live streaming different aspects of the process and a microphone were included in the setup (Figure 1).

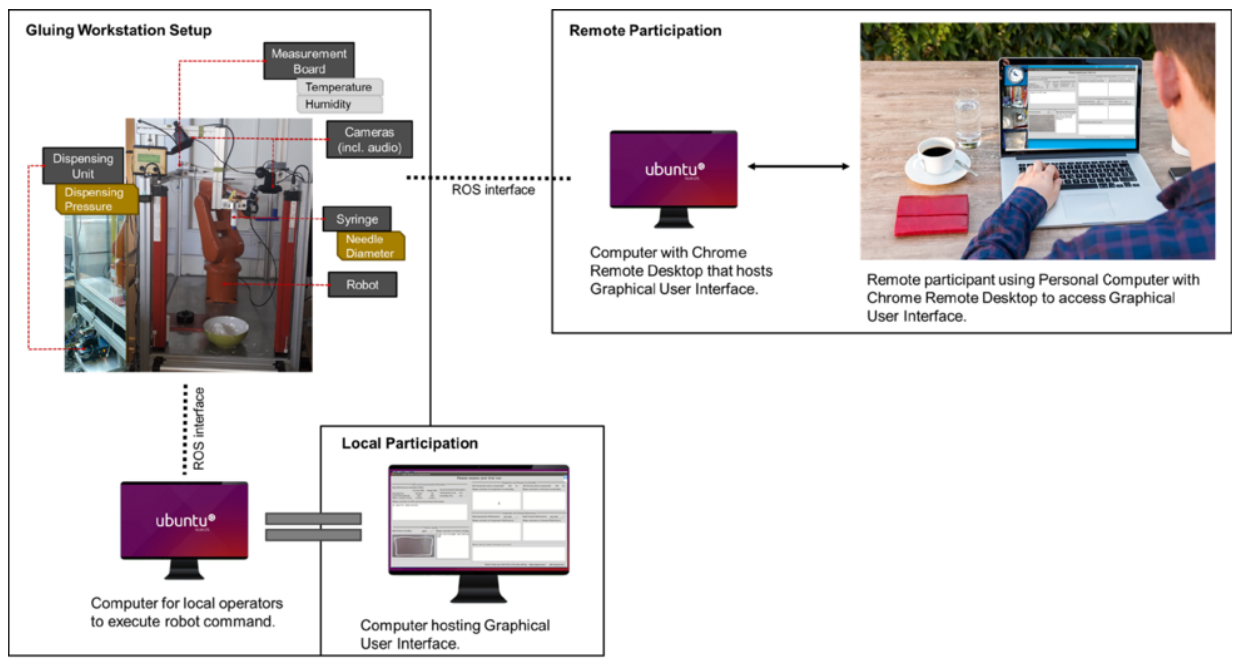

Figure 1. Overview of the setup for the robotised dispensing experiment.

\section{Results and Discussion}

Overall, 282 change-cycles related to the ramp-up process of this experiment were collected in this experiment from 16 participants, where 104 were conducted in Group A and 178 in Group B. To obtain the perceived workload estimates for the ramp-up experience during this experience and help gauge the proposed decision-support's usability and effectiveness from the participants' perspective, the widely accepted and used NASA-Task Load indeX (NASA-TLX) [4] was applied in its raw (or unweighted) form. The index takes into consideration the six different subscale categories mental demand, physical demand, temporal demand, performance, effort, and frustration, which are scored by the individual directly after conducting the task on a scale from 0 (good) to 20 (poor) for performance and 0 (low) to 20 (high) for the other categories. These are valuable insights that cannot necessarily be obtained through the other collected experimental data. However, using this measure is also not completely without disadvantages as participants may not fully recall their experience. Figure $\mathbf{2}$ shows the averaged results for the individual subscales across the different validation scenarios.

As was expected, the physical demand and effort are lower for the remote participants than they were for the local participants. This might also link to the temporal demand, which was higher in the local group as well. The mental demand was surprisingly deemed higher in the group of remote participants. This high mental demand 
might occur due to the need of remote participants to be engaged in the experiment. The individual's own performance was specifically well rated for participants in local participation in comparison to the remote group. This could be due to the fact that the local operators have a better feedback about the product and feel more immersed in the environment.

The highest average across the different scales was achieved in the frustration category and in particular for participants in Group A. This could be explained by the inperson participation for this group, where participants had to do the physical changes to the system themselves. Averaging the different subscales for the various groups provides $\sigma=11.08$ and $\sigma=7.8$ for Group A and B, respectively. As expected, this shows that the perceived workload was overall less for the remote participants, while the local participants felt they experienced the highest workload based on their results.

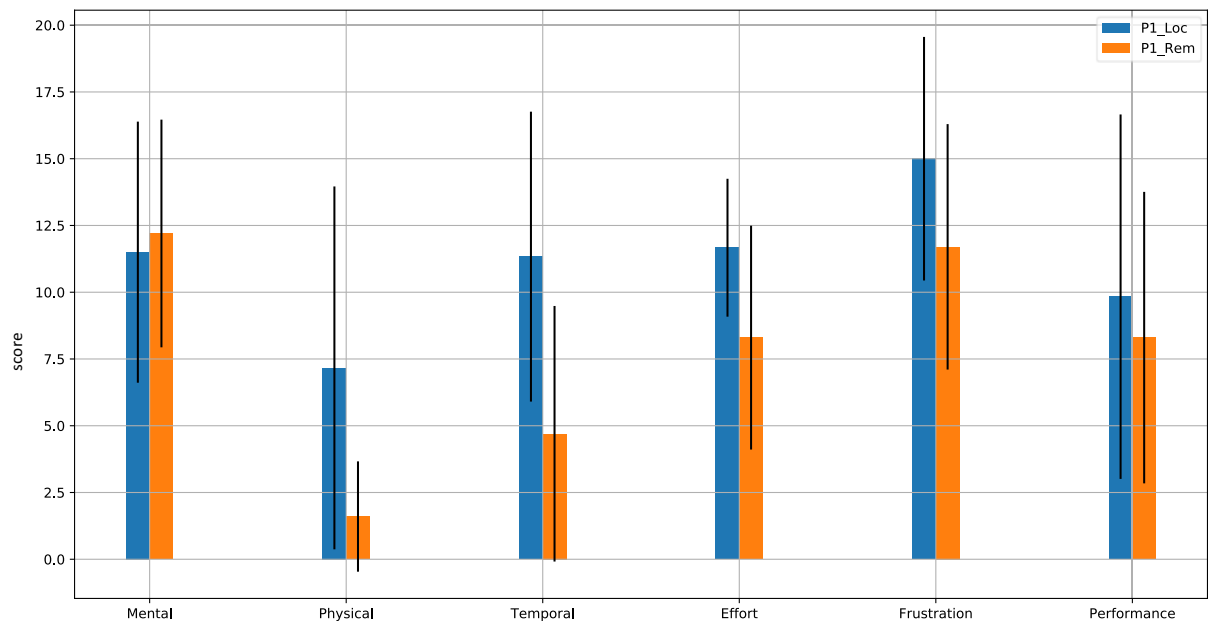

Figure 2. Average scores with standard deviation for subcategories of raw NASA-TLX for both groups.

\section{Conclusions and Future Work}

In this paper, the perceived mental workload for a robotised dispensing process in a local as well as remote setting was investigated using the raw NASA-TLX. Results showed that the perceived workload was overall lower for remote participants. Surprisingly, the perceived mental demand for remote participants was higher while the perceived performance was also rated lower compared to the local participants. However, literature might provide an explanation: As Cain [7] states, despite the difficulties of retaining focus in monitoring tasks, workload can be seen as modest. Thus, while well known, the disconnect between workload and efficiency remains poorly understood.

Using the perceived workload as a measure can highlight certain issues in terms of the operator's physiology or safety but applying self-reflective metrics such as the NASA-TLX are not time-sensitive for time-critical operations. Thus, including data from eye movements or heart rate can be promising approaches, which will be further investigated as part of this work in the future. Additionally, the paradigm of Industry 4.0 incorporates a transfer towards intelligent operations [17], in which artificial intelligence, 
robotics and automation enhance the human capabilities and counteract their weaknesses. This will facilitate the processes to have higher levels of safety, enhanced productivity and reduced mental load of human operators, which are fundamental elements for more intelligent workplaces. Furthermore, such technologies can be used to enable users to interact with robots remotely. In this case, the human operator would perform an industrial task a certain number of times for the robot then to carry out the task autonomously.

\section{Acknowledgements}

The authors gratefully acknowledge the financial support of the Engineering and Physical Sciences Research Council (EPSRC) Centre for Doctoral Training in Embedded Intelligence (EP/L014998/1) and "Digital Toolkit for optimisation of operators and technology in manufacturing partnerships” (DigiTOP) (EP/R032718/1).

\section{References}

[1] B. Wang, Y. Liu, J. Qian, and S. K. Parker, "Achieving Effective Remote Working During the COVID19 Pandemic : A Work Design Perspective,” vol. 70, no. 1, pp. 16-59, 2021, doi: 10.1111/apps.12290.

[2] M. S. Young, K. A. Brookhuis, C. D. Wickens, and P. A. Hancock, "State of science: mental workload in ergonomics," Ergonomics, vol. 58, no. 1, pp. 1-17, 2015, doi: 10.1080/00140139.2014.956151.

[3] B. H. Kantowitz, "Attention and Mental Workload," Proc. Hum. Factors Ergon. Soc. Annu. Meet., vol. 44, no. 21, pp. 456-459, 2000, doi: https://doi.org/10.1177/154193120004402121.

[4] S. G. Hart and L. E. Staveland, "Development of NASA-TLX (Task Load Index): Results of Empirical and Theoretical Research," in Human Mental Workload, vol. 52, P. A. Hancock and N. B. T.-A. in P. Meshkati, Eds. North-Holland, 1988, pp. 139-183.

[5] ILO Encyclopaedia of Occupational Health and Safety, "Mental Workload." https://www.iloencyclopaedia.org/k2-feed1/item/628-mental-workload (accessed Apr. 09, 2021).

[6] B. M. Huey and C. D. Wickens, Eds., Workload Transition - Implications for Individual and Team Performance. Washington, DC: National Academy Press, 1993.

[7] B. Cain, “A Review of the Mental Workload Literature," Def. Res. Dev. Toronto, no. 1998, pp. 4-1-4-34, 2007, [Online].

[8] R. J. Lysaght et al., "Operator Workload: Comprehensive Review and Evaluation of Operator Workload Methodologies," 1989.

[9] N. Stanton, A. Hedge, K. Brookhuis, E. Salas, and H. Hendrick, Eds., Handbook of Human Factors and Ergonomics Methods. CRC Press, 2005.

[10] S. Rubio, E. Díaz, J. Martín, and J. M. Puente, "Evaluation of Subjective Mental Workload: A Comparison of SWAT, NASA-TLX, and Workload Profile Methods," Appl. Psychol., vol. 53, no. 1, pp. 61-86, 2004.

[11]P. Gurevich, J. Lanir, and B. Cohen, Design and Implementation of TeleAdvisor: a Projection-Based Augmented Reality System for Remote Collaboration, vol. 24, no. 6. 2015.

[12] N. V. Flor, "Side-by-side collaboration : a case study," Int. J. Human-Computer Stud., vol. 49, pp. 201$222,1998$.

[13] J. C. Tang, "Findings from observational studies of collaborative work," Int. J. Man-Machine Stud., vol. 34, pp. 143-160, 1991.

[14] R. E. Kraut, S. R. Fussell, and J. Siegel, "Visual Information as a Conversational Resource in Collaborative Physical Tasks," Human-Computer Interact., vol. 18, pp. 13-49, 2003, doi: 10.1207/S15327051HCI1812.

[15]D. Gergle, R. E. Kraut, and S. R. Fussell, "Using Visual Information for Grounding and Awareness in Collaborative Tasks,” Human-Computer Interact., vol. 28, no. 1, pp. 1-39, Jan. 2013.

[16] S. R. Fussell, L. D. Setlock, J. Yang, E. Mauer, and A. D. I. Kramer, "Gestures Over Video Streams to Support Remote Collaboration on Physical Tasks,” Human-Computer Interact., vol. 19, pp. 273-309, 2004.

[17] K. Thoben, S. Wiesner, and T. Wuest, “'Industrie 4.0' and Smart Manufacturing - A Review of Research Issues and Application Examples,” Int. J. Autom. Technol., pp. 4-16, 2017. 